\title{
Study on Regional Scientific and Technological Innovation Platform Innovations Evaluation Based on BP Neural Network
}

\author{
Zhang Liyan \\ Harbin University of Science and Technology, school of Economics \\ 13684601379@163.com
}

\begin{abstract}
Based on the needs of the Regional Scientific and Technological Innovation Platform, innovations evaluation system was established by AHP. In order to simulate the experts' experiences and thinking, we used the improved BP neural network model. After training by putting in actual data, the improved BP neural network model was put in use to evaluate and manage the innovations created by the Regional Scientific and Technological Innovation Platform.
\end{abstract}

Keywords: regional scientific and technological innovation platform; BP neural network; innovations evaluation

\section{Introduction}

With the accelerated process of economic globalization, the world economy appears regional characteristics obviously. In the rapid development of science and technology, the idea that innovation is a key factor in promoting economic growth has become the consensus by academia, enterprises and the government. Faced with intense competition in international technology and economy, most of developed countries have built firstclass scientific and technological innovation platform as a support for innovative activity preferences and achieve leapfrog development of strategic initiatives.

Regional scientific and technological innovation platform is established in the common needs of pivotal technology innovation in the relative industrial clusters, hunting, gathering and integration knowledge, information, technology, and other related resources for innovation. And provide each innovation subject in industrial clusters with public services and industrial technology innovation support by improving the liquidity, diffusibility of innovations in the platform. In a conclusion, regional scientific and technological innovation platform is an aggregate which linking the various nodes of innovation innovative needs and services effectively [1].Technological innovation platform, as an important carrier of innovation activities in the whole economic society, plays an important role in the regional science and technology and economic development. Thus in the present international science and technology and economic environment, a scientific theory and methodology is necessary to evaluate innovations which was created by enterprises in the innovation platform. It can not only test the efficiency of innovation in the platform, but also provide enterprises innovative resources in the future more efficiently.

Nowadays, two important issues must be faced in the evaluation in the platform. First, how the quantitative results can be obtained as quickly as possible in the evaluation on a large number of innovations created by enterprises in the platform continually. Second, each evaluation result of innovations must be scientific, reasonable and consistent with the thinking of experts. These two issues are difficult to simultaneously solve by the general evaluation methods and expert evaluation group. 
To use new method which combines BP neural networks with a computer simulation of experts' experience and thinking in the evaluation can just satisfy to two key areas of scientific and technological innovation platform innovations quantitative evaluation requirements. Thus it is possible to the quantitative evaluation of regional technological innovation platform innovations. Using improved BP neural network intelligent innovation evaluation methods to simulate expert thought according to the regional scientific and technological innovation platform innovations evaluation features, is the research in this paper.

\section{Establish Evaluation index System of Regional Scientific and Technological Innovation Platform Innovations}

Advanced scientific evaluation system is a key for Regional Innovation platform innovations to achieve quantitative evaluation, and scientific and effective, practical and feasible, comparable and flexible should be the feature of the evaluation system [2]. Thus we looked for the main factors that could effectively reflect innovation and state of the essential characteristics of systems analysis on the basis of characteristics of innovations. Then we combined domestic and foreign indicators related to industrial science and technology, and get four main factors. They are: the innovation skills, Innovations investment level in science and technology, Diffusion of innovation capabilities and value-added level of innovation[3].

According to the AHP theory, we made the consistency test for all levels of each judgment matrix, to meet the requirements for consistency matrix, As each judgment matrix consistency proportion C.R. ${ }_{c}^{1}=0.0038, C . R .{ }_{c}^{2}=3.8973 \times 10^{-4} \ldots \ldots$. Using the geometric average method to determine the value of the relevant experts conduct a comprehensive, After criteria layer index weights calculated evaluation system innovations weight $w_{b_{1}}^{a}=0.5000, w_{b_{2}}^{a}=0.1566, w_{b_{3}}^{a}=0.1111, w_{b_{4}}^{a}=0.2323$,

Index layer relative weights is $w_{c_{1}}^{b_{1}}=0.1758, \quad w_{c_{2}}^{b_{1}}=0.2215$, $w_{c_{3}}^{b_{1}}=0.3730, w_{c_{4}}^{b_{1}}=0.2297, \ldots \ldots, w_{c_{8}}^{b_{4}}=1$ 。

Comprehensive evaluation index weights are $w_{c_{1}}^{a}=0.0879, w_{c_{2}}^{a}=0.1108$, $w_{c_{3}}^{a}=0.1865, \ldots \ldots w_{c_{8}}^{a}=0.2323$ 。

According to comprehensive weight, the order of indexes on the two layers(Criterion layer and Index layer) are: $\mathrm{B}_{1}, \mathrm{~B}_{4}, \mathrm{~B}_{2}, \mathrm{~B}_{3}$ and $\mathrm{C}_{8}, \mathrm{C}_{3}, \mathrm{C}_{4}, \mathrm{C}_{7}$, $\mathrm{C}_{2}, \mathrm{C}_{5}, \mathrm{C}_{1}, \mathrm{C}_{6}$ 。 Innovation and achievement evaluation system established as Figure 1 .

\section{BP Artificial Neural Network Training}

\subsection{Determine the Evaluation of Neural Network Training Samples}

This paper according to actual needs of the Heilongjiang Provincial Science and Technology Innovation Platform. The innovations are selected from the hightechnology field"040000 mechanical and electronical engineering" categories of products in the field of " 040100 Automation machinery and equipment" class products "040101 advanced manufacturing equipment and installations"20 categories of innovation as a quantitative evaluation of typical products ,After the dimensionless and standardized treatment of actual data on the innovation survey, 20 innovations code and eight evaluation indexes as shown in Table 1. 


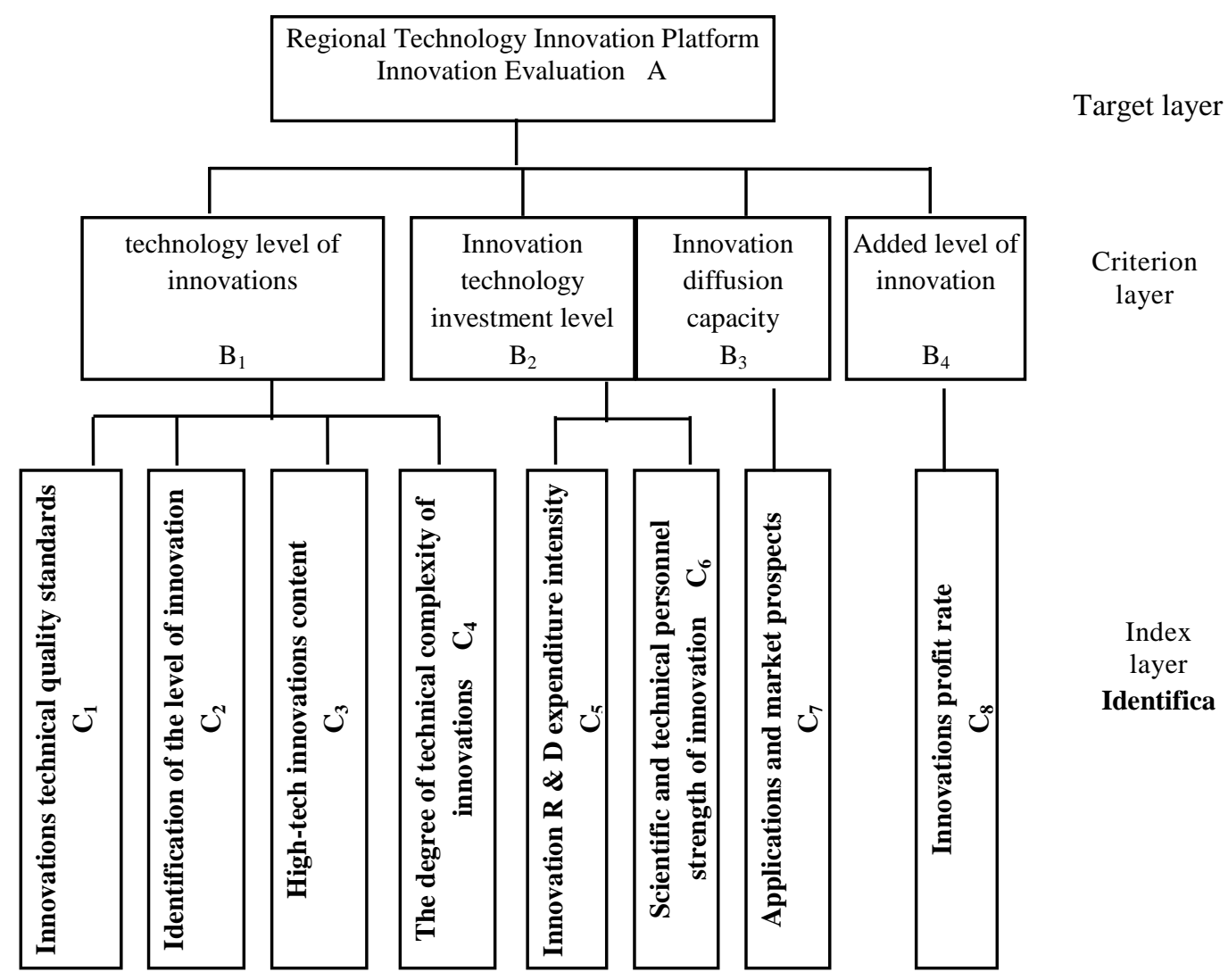

Figure 1. Model of Innovation and Achievement Evaluation System

Table 1. The Value of Evaluation Indexes

\begin{tabular}{c|c|c|c|c|c|c|c|c|c}
\hline No. & Specimen code & $\mathrm{C}_{1}$ & $\mathrm{C}_{2}$ & $\mathrm{C}_{3}$ & $\mathrm{C}_{4}$ & $\mathrm{C}_{5}$ & $\mathrm{C}_{6}$ & $\mathrm{C}_{7}$ & $\mathrm{C}_{8}$ \\
\hline 1 & $040101-001$ & 0.85 & 0.90 & 0.80 & 0.70 & 0.85 & 0.82 & 0.90 & 0.60 \\
\hline 2 & $040101-002$ & 0.95 & 0.90 & 0.90 & 0.80 & 0.95 & 1.00 & 0.95 & 0.83 \\
\hline 3 & $040101-003$ & 0.70 & 0.80 & 0.75 & 0.60 & 0.70 & 0.58 & 0.75 & 0.63 \\
\hline 4 & $040101-004$ & 0.70 & 0.70 & 0.60 & 0.60 & 0.62 & 0.69 & 0.80 & 0.50 \\
\hline 5 & $040101-005$ & 0.70 & 0.70 & 0.60 & 0.65 & 0.53 & 0.75 & 0.68 & 0.42 \\
\hline 6 & $040101-006$ & 0.95 & 0.90 & 0.95 & 0.90 & 0.90 & 1.00 & 0.86 & 1.00 \\
\hline 7 & $040101-007$ & 0.95 & 0.85 & 0.90 & 0.90 & 0.95 & 1.00 & 0.50 & 0.93 \\
\hline$\cdots$ & $\ldots$ & & & & & & & & \\
\hline 19 & $040101-019$ & 0.85 & 0.70 & 0.70 & 0.90 & 0.80 & 0.73 & 0.73 & 0.42 \\
\hline 20 & $040101-020$ & 0.85 & 0.70 & 0.70 & 0.55 & 0.75 & 0.39 & 0.40 & 0.33 \\
\hline
\end{tabular}

\subsection{BP Network Training}

Back Propagation is error back propagation neural network, a type of network most commonly used It's able to achieve mapping transformation feed forward networks. It is a typical error correction method. It has the ability to theoretically 
approximate any nonlinear continuous function, as well as simple structure and it's also easy to program. It has been widely used in many areas Because of this backpropagation algorithm based on forward multilayer network uses a steepest descent optimization algorithm, Convergence of the algorithm is very slow, ordinarily it requires thousands of or even more iteration to meet the convergence requirements. And it's easy to fall into local minima. To this end, there have been many improvements to the algorithm. In order to improve the training speed, avoid falling into local minimum and improve generalization ability, It is combined the method of momentum and adaptive learning rate in this paper.

In order to prevent the network into a local minimum, the additional momentum method used is based on the back-propagation method in every change of weight plus a weight proportional to the value of the previous amount of change, And in accordance with back propagation method to produce a new weight change. The formula with additional momentum factor value adjustment is:

$$
\left\{\begin{array}{l}
\Delta w_{i j}(k+1)=(1-m c) \eta \delta_{i} x_{j}+m c \Delta w_{i j}(k) \\
\Delta b_{i}(k+1)=(1-m c) \eta \delta_{i}+m c \Delta b_{i}(k)
\end{array}\right.
$$

Among them, $\mathrm{K}$ is training times, $\Delta w$ is incremental weights, $\eta$ is learning rate; $\delta$ is the Error; $\mathrm{x}$ is network input; $\mathrm{mc}$ is momentum factor, generally takes about 0.9 .

For a specific problem, It is difficult. to select the appropriate learning rate .Therefore, it's useful to adopt automatic adjustment method of learning rate in network training, Namely adaptive learning rate method. Guidelines for the application of the method are: Check the weights of correction value whether really reduces the error function, If so, it improves that the value of the selected learning rate is small, you can increase an amount; If not, it improves the produced are overshoot, Then you should reduce the rate value of the learning. The following formula gives the adjustment formula of an adaptive learning rate:

$$
\begin{gathered}
\eta(k+1)= \begin{cases}1.05 \eta(k) & \operatorname{SSE}(k+1)<\operatorname{SSE}(k) \\
0.7 \eta(k) & \operatorname{SSE}(k+1)>1.04 \operatorname{SSE}(k) \\
\eta(k) & \text { others }\end{cases} \\
\quad \operatorname{SSE}=\sum_{i=1}^{n}\left(y_{i}-y_{i}^{\prime}\right)^{2} \quad(i=1, \cdots, n)
\end{gathered}
$$

Among them, $\eta$ is Learning rate; $\mathrm{K}$ is Training times; SSE is Error function; $y_{i}$ is Learn output samples; $y_{i}^{\prime}$ is the actual output value of the network after training; $n$ is the number of learning samples ${ }^{[5]}$ 。

It is used a two-story BP neural network in this paper, It takes 20 neurons in hidden layer.,uses hyperbolic tangent activation function and linear output layer activation function. The network has eight input nodes and an output node, As are shown in Figure 2. 


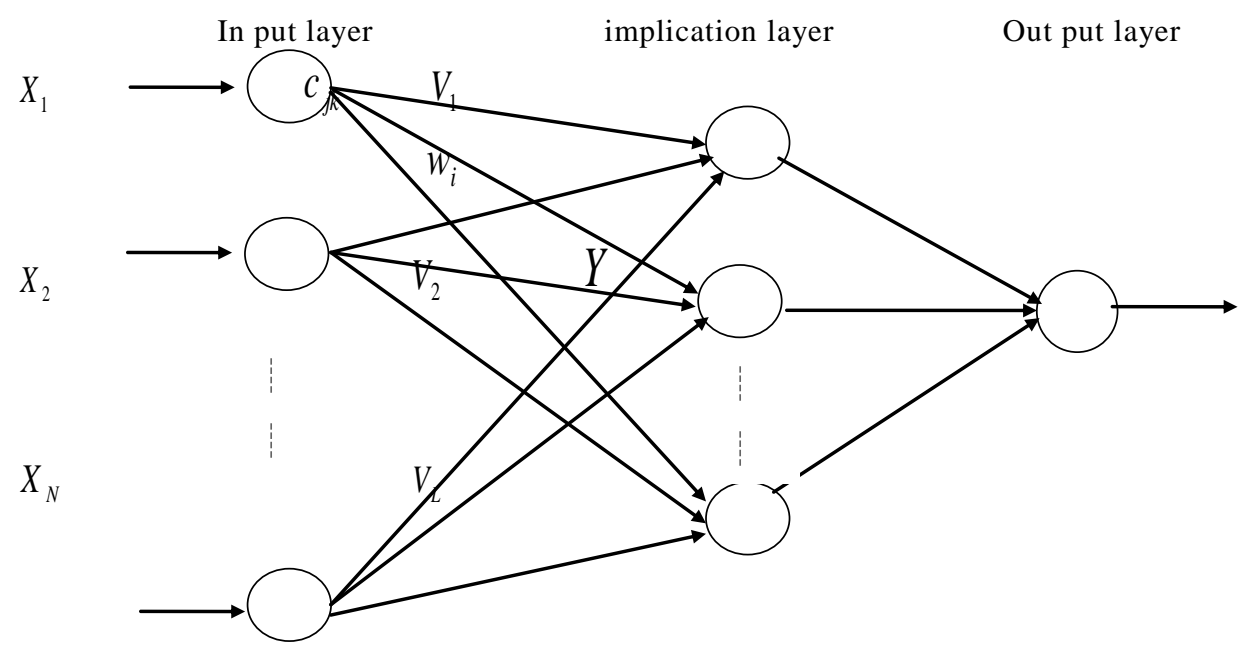

Figure 2. Regional Science and Technology Innovation Platform, Innovation Evaluation of BP Network Structure

(1)Initialization. The network weights $\left(w_{i j}^{1}, w_{j}^{2}\right)$ and The value of the threshold $\left.b_{i}^{1}, b^{2}\right)$ is the random number between $(-1,1) . w_{i j}^{1} b_{i}^{1}$ is the first layer weights and thresholds network connection weights. $w_{j}^{2} b^{2}$ is the second layer weights and thresholds of the network.

(2)BP network training. Using linear weighted sum method and attribute comprehensive evaluation method, Combined with expert's experience and ideas on innovation typical of Table 1 (sample) get on a comprehensive evaluation of the true value(See Table 2).BP network training use function train bpx. $m$ in Neural Network Toolbox of MATLAB Language. When the train reaches the maximum number of training, or the network squared error is reduced to below the expected error, Network stops training, And get the final weights and thresholds $\left(w_{i j}^{1}, b_{i}^{1}, w_{j}^{2}, b^{2}\right)$.The whole design and training is in MATLAB environment, using the computer, and training set is 20 samples, Expected error is 0.000001. When the number of cycles is up to15000, it achieves precision, BP network training time is 280 seconds. Using BP networks tests on innovation evaluation. The results are shown in Table 2.

Table 2. The Evaluation Value of Specimen

\begin{tabular}{c|c|c|c|c}
\hline No. & Specimen code & $\begin{array}{c}\text { The true value of } \\
\text { specimen }\end{array}$ & BP out put value & BP 误差 \\
\hline 1 & $040101-001$ & 0.775023 & 0.77628 & -0.0013 \\
\hline 2 & $040101-002$ & 0.892459 & 0.89575 & -0.0033 \\
\hline 3 & $040101-003$ & 0.692411 & 0.69261 & -0.0002 \\
\hline 4 & $040101-004$ & 0.625380 & 0.62116 & 0.0042 \\
\hline 5 & $040101-005$ & 0.592370 & 0.59176 & 0.0006 \\
\hline 6 & $040101-006$ & 0.937346 & 0.93985 & -0.0025 \\
\hline 7 & $040101-007$ & 0.871634 & 0.87190 & -0.0003 \\
\hline$\ldots$ & $\ldots$ & & & \\
\hline 19 & $040101-019$ & 0.686706 & 0.68519 & 0.0015 \\
\hline 20 & $040101-020$ & 0.567090 & 0.56668 & 0.0004 \\
\hline
\end{tabular}


The trained BP neural network in the first layer of the network connection weights (160):

$$
\begin{gathered}
w_{11}^{1}=0.90626, \quad w_{12}^{1}=-0.89195, \quad w_{13}^{1}=0.55808, \quad w_{14}^{1}=-0.031308, \quad w_{15}^{1}=0.6127, \\
w_{16}^{1}=0.10066, \quad w_{17}^{1}=-0.040608, \quad w_{18}^{1}=0.42021, \ldots \ldots . \ldots ., w_{208}^{1}=0.18131 。
\end{gathered}
$$

The second layer of the network connection weights weights(20): $w_{1}^{2}=-0.72718, w_{2}^{2}=-$ 0.11817 ,

$$
w_{3}^{2}=0.28284, \quad w_{4}^{2}=0.72973, \quad w_{5}^{2}=-0.2338, \ldots \ldots . \ldots, \quad w_{20}^{2}=0.93454 。
$$

The threshold of the first layer of the network(20): $b_{1}^{1}=0.23126, b_{2}^{1}=-0.96627$, $b_{3}^{1}=-0.90912, \quad b_{4}^{1}=-0.68338, \quad b_{5}^{1}=0.25529, \quad b_{6}^{1}=-0.88244, \ldots \ldots, b_{20}^{1}=0.20187$ 。

The threshold of the second layer of the network $b^{2}=-0.10776$ 。

\section{The Application of BP Neural Network in the Regional Technological Innovation Platform Innovations Evaluation}

In the actual practice of Heilongjiang Province Science and Technology Innovation Platform Innovation Evaluation management, Heilongjiang Provincial Science and Technology Department regularly organizes the experts related to high-technology and management to evaluate. Relevant innovations It is based on regional science and technology innovation platform evaluation method innovations of improved BP neural network in this paper, to complete evaluation of the relevant regional technological innovation platform innovations. For this purpose it selected 10 innovations that belong to the catalog of "040101 Advanced manufacturing equipment and installations" innovations evaluate to test.

First, innovations indicator data were normalized, and we get the actual measured value of each index, As shown in Table 3. Next we input the measured value of each index into the trained BP network connection weights and each threshold to the BP neural network, Calculated innovations evaluation results, As shown in Table 3.

Table 3. Normalized Innovations Value and Evaluation Level

\begin{tabular}{c|c|c|c|c|c|c|c|c|c|c}
\hline No. & $\begin{array}{c}\text { Innovations } \\
\text { code }\end{array}$ & $\mathrm{C}_{1}$ & $\mathrm{C}_{2}$ & $\mathrm{C}_{3}$ & $\mathrm{C}_{4}$ & $\mathrm{C}_{5}$ & $\mathrm{C}_{6}$ & $\mathrm{C}_{7}$ & $\mathrm{C}_{8}$ & $\begin{array}{c}\text { Evaluation } \\
\text { level }\end{array}$ \\
\hline 1 & $040101-001$ & 0.90 & 0.73 & 0.71 & 0.66 & 0.60 & 0.63 & 0.40 & 0.60 & $\mathrm{~B}$ \\
\hline 2 & $040101-002$ & 0.95 & 0.93 & 0.80 & 0.75 & 0.94 & 0.90 & 0.50 & 0.31 & $\mathrm{~A}$ \\
\hline 3 & $040101-003$ & 0.96 & 0.92 & 0.81 & 0.70 & 0.29 & 0.78 & 0.60 & 0.26 & $\mathrm{~B}$ \\
\hline 4 & $040101-004$ & 0.85 & 0.71 & 0.60 & 0.52 & 0.26 & 0.48 & 0.35 & 0.23 & $\mathrm{D}$ \\
\hline 5 & $040101-005$ & 0.85 & 0.88 & 0.65 & 0.63 & 0.06 & 0.20 & 0.61 & 0.20 & $\mathrm{C}$ \\
\hline 6 & $040101-006$ & 0.72 & 0.76 & 0.65 & 0.40 & 0.09 & 0.38 & 0.30 & 0.03 & $\mathrm{D}$ \\
\hline 7 & $040101-007$ & 0.70 & 0.68 & 0.70 & 0.60 & 0.05 & 0.65 & 0.30 & 0.22 & $\mathrm{C}$ \\
\hline 8 & $040101-008$ & 0.90 & 0.89 & 0.73 & 0.65 & 0.14 & 0.53 & 0.56 & 0.40 & $\mathrm{~B}$ \\
\hline 9 & $040101-009$ & 0.93 & 0.90 & 0.72 & 0.55 & 0.57 & 0.58 & 0.30 & 0.38 & $\mathrm{C}$ \\
\hline 10 & $040101-010$ & 0.72 & 0.65 & 0.55 & 0.50 & 0.17 & 0.45 & 0.30 & 0.18 & $\mathrm{D}$ \\
\hline
\end{tabular}

Compared the evaluation of the test results and the true value from the innovation expert evaluation of the test results and errors are shown in Table 4.Typical results 
innovations error is less than a given error, It proves that the evaluation method proposed in this paper has good application effect

Table 4. Innovations Result and Error

\begin{tabular}{c|c|c|c|c}
\hline No. & Innovations code & True value & Calculate value & error \\
\hline 1 & 040101001 & 0.647410 & 0.649332 & -0.001922056 \\
\hline 2 & 040101002 & 0.694680 & 0.689476 & 0.005203548 \\
\hline 3 & 040101003 & 0.613933 & 0.63674 & -0.022806868 \\
\hline 4 & 040101004 & 0.468657 & 0.472306 & -0.00364872 \\
\hline 5 & 040101005 & 0.493949 & 0.509171 & -0.015222362 \\
\hline 6 & 040101006 & 0.383070 & 0.405208 & -0.022137546 \\
\hline 7 & 040101007 & 0.457610 & 0.477107 & -0.019497308 \\
\hline 8 & 040101008 & 0.584423 & 0.6058 & -0.021377295 \\
\hline 10 & 040101009 & 0.590237 & 0.58946 & 0.000776895 \\
\hline
\end{tabular}

\section{Conclusions}

This article goes from the actual needs of regional scientific and technological innovation platform management, to establish a regional science and technology innovation platform evaluation index system of innovation, to use improved BP neural network model for a typical data innovation training, After the test of evaluation ,part of the innovation, the results show that the proposed intelligent evaluation method has performance of scientific and practical. This method combined with rule-based evaluation method can provide an effective means for government high-tech industry manage and evaluate the regional scientific and technological innovation platform.

\section{Acknowledgements}

The research work was supported by Scientific project of Heilongjiang education department (No. 12541179) , Study on regional scientific and technological innovation platform innovations evaluation.

\section{References}

[1] Fredman, "Economics on Industrial innovation", Beijing University Press, (2004), pp. 289-298

[2] D. You and W. Yang, "Structure of evaluation indexes of High-tech enterprises", innovation ability, Technology Economics, vol. 8, (2003), pp.53-55.

[3] H. Wang and Y. Hu, "Study on quantitative identify indexes system of High-tech products", Scientific Research Management, vol. 1, (2003), pp.116-122.

[4] G. Liu, C. Huang and J. Ding, "BP neural network model on water quality evaluation", Chinese environment Science, vol. 18, no. 6, (1998), pp. 514-517.

[5] T. Yang, "Research on the High-tech enterprises' evaluation by BP neural network", Chinese soft science, vol. 5, (2003), pp. 96-98.

[6] C. Gao, D. Peng and Y. Zhong, "Research on Automatic Comprehensive Evaluation Model Selection", Journal of Harbin University of Science and Technology, vol. 16, no. 6, (2011), pp.118-123

[7] H. Zhao and Q. Li, "The application of Fuzzy Mathematical Method to the Evaluation of Grain Production”, Journal of Harbin University of Science and Technology, vol. 19, no. 4, (2014), pp. 79-83 
International Journal of $u-$ and e- Service, Science and Technology Vol.9, No. 1 (2016) 